\title{
Recall of disclosed Apolipoprotein E genotype and lifetime risk estimate for Alzheimer's disease: The REVEAL Study
}

Susan LaRusse Eckert, $M S^{1}$, Heather Katzen, PhD ${ }^{2,3}$, J. Scott Roberts, PhD ${ }^{4}$, Melissa Barber, MS ${ }^{5}$, Lisa D. Ravdin, PhD ${ }^{2}$, Norman R. Relkin, $M D$, $P h D^{2}$, Peter J. Whitehouse, $M D, P h D^{5,6}$, and Robert C. Green, $M D, M P H^{4,7}$

\begin{abstract}
Purpose: To determine whether individuals recall their apolipoprotein E genotype and numeric lifetime risk estimates after undergoing a risk assessment for Alzheimer's disease. Methods: One-hundred and four participants underwent Alzheimer's disease risk assessment that included disclosure of apolipoprotein E genotype and a numeric lifetime risk estimate. Results: At six weeks and one year post-disclosure, 59\% and $48 \%$ of participants, respectively, recalled their lifetime risk estimate, and $69 \%$ and $63 \%$ recalled their apolipoprotein $E$ genotype. Participants were more likely to remember their genotype than numeric lifetime risk estimate at one year $(P<$ 0.05). Apolipoprotein $\mathrm{E} \varepsilon 4$-positive participants had better recall of their genotype at both time points $(P<0.05)$. Participants were more likely to recall whether they carried the "risk-enhancing form of apolipoprotein $E$ " than their specific genotype $(P<0.05)$. Conclusions: These data suggest that apolipoprotein $\mathrm{E}$ genotype, especially the presence of an $\varepsilon 4$ allele, is more memorable than a numeric risk estimate for Alzheimer's disease. Participants recalled genotype information in a more simplified, binary form. Health professionals testing for complex disorders such as Alzheimer's disease must find an appropriate balance between communicating risk in an understandable format and addressing the probabilistic nature of the information. Genet Med 2006:8(12):746-751.
\end{abstract}

Key Words: genetic susceptibility testing, REVEAL, Alzheimer's disease, APOE, risk information recall, genetic counseling

Alzheimer's disease $(\mathrm{AD})$ is a complex late-onset disease known to have a substantial genetic component. ${ }^{1}$ There are three known genes associated with the early-onset autosomal dominant form of $\mathrm{AD}$, but these genes account for $<2 \%$ of $\mathrm{AD}$ cases. ${ }^{2}$ The polymorphism associated with the much more common late-onset $\mathrm{AD}$, the $\varepsilon 4$ allele of the apolipoprotein $\mathrm{E}$ (APOE) gene, ${ }^{3}$ is relatively common in the general population with approximately $25 \%$ of individuals carrying at least one $\varepsilon 4$ allele.

At the present time genetic testing for late-onset $\mathrm{AD}$ is not widely performed or recommended because of the low predictive value of $A P O E$ genotyping and the lack of proven preventative options. ${ }^{4-7}$ Genetic counseling and testing for complex disorders such as $\mathrm{AD}$ will become more widespread if addi-

\footnotetext{
From the ${ }^{1}$ Taub Institute for Research on Alzheimer's Disease and the Aging Brain, Columbia University, New York, New York; ${ }^{2}$ Department of Neurology and Neuroscience, Weill Medical College of Cornell University, New York, New York; ${ }^{3}$ Department of Neurology, University of Miami Miller School of Medicine, Miami, Florida; Departments of ${ }^{4}$ Neurology and ${ }^{7}$ Medicine (Genetics Program), Boston University School of MedicineBoston, Massachusetts; ${ }^{5}$ University Memory and Aging Center, Case Western Reserve University School of Medicine/ University Hospitals of Cleveland, Cleveland, Ohio; and ${ }^{6}$ Department for Biomedical Ethics, Case Western Reserve University School of Medicine, Cleveland, Ohio.
}

Susan LaRusse, 4 Kenneth Road, Montclair, NJ 07043.

Submitted for publication June 19, 2006.

Accepted for publication September 18, 2006.

DOI: 10.1097/01.gim.0000250197.44245.a3 tional susceptibility genes and effective preventative strategies are identified. A major aim of genetic counseling for complex disorders is to inform an individual of his or her probabilistic risk of developing a specific disease; therefore, an essential component of genetic counseling is risk communication. Risk perception and information recall are two measures that are commonly used to assess the effectiveness of risk communication. Although studies have explored perceived risk of $\mathrm{AD}$ in our study population, ${ }^{8,9}$ little is known about how people recall risk estimates that are associated with genetic susceptibility testing for common disorders such as $\mathrm{AD}$.

The vast majority of studies on risk information recall have been carried out in breast cancer genetic testing and counseling. The risk information presented in genetic counseling and testing for breast cancer is necessary for the health practitioner and patient to make informed medical decisions regarding appropriate risk management; this scenario may also apply to $\mathrm{AD}$ in the future if preventative strategies are identified. Studies examining the recall of risk information in breast cancer populations have shown that recall is poor and accuracy of risk recall varies widely. ${ }^{10-12}$ One reason recall accuracy varies in the literature is that investigators have used different stringency criteria to define accuracy. ${ }^{13}$ In addition, there is no consistency in the format in which risk figures are presented. ${ }^{14,15}$ Studies have likely used different formats to present risk figures because women have expressed different preferences in how 
risk information is presented. ${ }^{16} \mathrm{~A}$ consensus for the most effective method of communicating risk information has not been reached. There is a need for more research in this area, specifically on how to communicate complex risk information for common diseases. We present the first study on recall of risk information in genetic susceptibility testing for Alzheimer's disease.

The REVEAL Study (Risk Evaluation and Education for Alzheimer's Disease) is the first multi-center randomized controlled trial to evaluate the impact of a risk assessment program using APOE genotyping and disclosure for nondemented adult children of people diagnosed with AD. For this paper, we assessed the recall accuracy of participants who received both APOE genotype as well as a lifetime risk estimate of developing $\mathrm{AD}$. We hypothesized that 1) considering the amount of information presented in the study protocol, accurate recall of the specific genotype and numeric risk estimate would be poor with over $25 \%$ of the participants not able to accurately recall the information at one year; 2) based on previous REVEAL reports describing the impact of genetic testing on risk perception, ${ }^{8-9}$ genotype information would be more accurately recalled than lifetime risk information and; 3) participants would be more likely to recall their $\varepsilon 4$ status than their specific APOE genotype. Previous studies on hereditary breast cancer found that baseline worry about the disease, ${ }^{17}$ family history, ${ }^{18}$ and education ${ }^{19}$ were associated with both risk perception and recall, thus, we were also interested in determining whether these factors along with gender influenced accurate recall for an $\mathrm{AD}$ risk assessment.

\section{MATERIALS AND METHODS}

\section{Overview}

The REVEAL Study's methods have been described in detail in previous publications. ${ }^{8,9,20,21}$ Briefly, the study protocol was developed by a multidisciplinary team of experts in the fields of $\mathrm{AD}$, neurology, genetics, genetic counseling, psychology, and bioethics. Development of the protocol was overseen and approved by a study External Advisory Board, as well as institutional review boards at each of the three study sites. All participants gave written informed consent.

\section{Participants and procedures}

All REVEAL study participants were adult children of a living or deceased individual diagnosed with late-onset $\mathrm{AD}$ (age of onset $\geq 60$ years) and were referred to the study through systematic ascertainment from $\mathrm{AD}$ research registries or self-referral. All participants underwent neuropsychological screening and were judged to be cognitively intact. Participants were randomized to either an Intervention or Control Arm in a 2:1 ratio, respectively, after blood was drawn for genetic testing. Participants in the Intervention Arm received both $A P O E$ genotype and a lifetime risk estimate based on family history, genotype, and gender. The Control Arm did not learn their $A P O E$ genotype but did receive a lifetime risk estimate based on gender and family history. For the purposes of this paper, we focused on information recall among the Intervention Arm participants $(\mathrm{N}=104)$ only because we were interested in comparing correct recall of $A P O E$ genotype and a numeric lifetime risk estimate.

The study protocol consisted of five stages: 1) A telephone interview in which demographic and family history information was obtained; 2) a group education session which provided a description of the study, an overview of genetic principles and the genetics of $\mathrm{AD} ; 3)$ a blood drawing session in which neuropsychological screening, specifically the Repeatable Battery for the Assessment of Neuropsychological Status (RBANS $)^{22}$ was administered along with individualized genetic counseling; 4) a disclosure session in which a risk assessment was provided as well as APOE genotype; and 5) follow-up sessions at six weeks, six months and one year post-disclosure in which questionnaires were self-administered. In an effort to focus on initial and long-term recall of the information, we analyzed questionnaire responses from the six-week and oneyear post-disclosure sessions.

\section{Information provided during counseling}

During the educational session, the genetic counselors provided information about the study protocol, $\mathrm{AD}$, and genetics via a standardized slide show. A printout of the presentation was given to each participant. The genetic counselors presented statistics on the prevalence of $\mathrm{AD}$ as well the general population lifetime risk of developing $\mathrm{AD}$. An overview of genetic principles was presented, and the genetic counselors discussed familial $\mathrm{AD}$ and the associated genes. A significant portion of the educational session was devoted to APOE: specifically, the three different alleles, the six possible genotypes, the frequency of $A P O E$ genotypes in the general population, and statistics to demonstrate that the $\varepsilon 4$ allele is a susceptibility gene that is neither sufficient nor necessary to cause AD. Participants had the opportunity to ask questions about the information presented during the educational session and during the individualized genetic counseling session.

During the disclosure session, participants in the Intervention Arm received three pieces of risk information: A lifetime risk estimate which was defined as the risk from birth to age 85; a remaining risk estimate which was defined as the risk from the participant's current age to age 85; and their APOE genotype. Participants were also informed of the general population lifetime risk of developing $\mathrm{AD}$ as well as risk to all first-degree relatives for comparative purposes. The lifetime risk estimates ranged from $13 \%$ to $57 \%$ depending on an individual's gender and $A P O E$ genotype. Risk estimates were formulated using data from a multi-center AD genetic epidemiology studies based at Boston University. ${ }^{21}$ The risk estimates were presented in oral, visual, and written formats and included in a take-home letter provided to participants. APOE genotype was not provided in the letter in order to minimize the possibility of genetic discrimination by disclosure to an unintended third party. The risk estimate was presented as a percentage and although it was stressed that an individual's specific risk may vary according to other unknown risk factors, a range of risk 
was not offered. Risk curves tailored to a participant's genotype and gender were generated. The risk curves included a line representing the general population lifetime risk, a line representing risk to all first-degree relatives, and a line representing the individual's lifetime risk based on family history, gender, and genotype (Fig. 1). The risk curves were not taken home by the participants.

\section{Measures}

At six weeks and twelve months after disclosure, participants were asked a series of written survey questions designed to assess their perceptions of $\mathrm{AD}$ risk, the impact of the risk and genotype information, and their recollection of the information presented during the disclosure session. All questionnaires were self-administered onsite, although in rare instances, the questionnaire was mailed to the participant if he or she was unable to come to the site. Questions regarding a participant's information recall at six weeks post-disclosure were as follows (response options in parentheses): 1) "What is the percentage you were given as your lifetime risk of developing AD?" (open-ended); 2) "What were your APOE genetic test results?" (I don't remember, $\varepsilon 2 / \varepsilon 2, \varepsilon 2 / \varepsilon 3, \varepsilon 2 / \varepsilon 4, \varepsilon 3 / 3 \varepsilon, \varepsilon 3 / \varepsilon 4$, $\varepsilon 4 / \varepsilon 4)$. At one year post-disclosure, participants were asked the above questions as well as the following: 1) "What form (allele) of $A P O E$ is the risk-enhancing form?" ( $\varepsilon 2, \varepsilon 3, \varepsilon 4$, I don't remember); 2) "Do you have the risk-enhancing form (allele) of APOE?" (Yes: one copy, Yes: two copies, No, I don't remember).

\section{Data analyses}

Descriptive statistics were used to characterize the sample in terms of its demographics and information recall. Responses were analyzed to examine the extent to which participants recalled lifetime risk information and genotype at six weeks and at one year following the disclosure session. Based on definitions of recall accuracy in previous reports, ${ }^{11,14,23}$ some leeway was given in determining accurate recall of lifetime risk, which we defined as within five percentage points of the estimate provided during the disclosure section. Accurate recall of genotype was defined as the correct recall of both $A P O E$ alleles. McNemar's $\chi^{2}$ analysis was employed to compare the accuracy of recall of specific genotype and numeric risk estimates. Correlative and regression analyses were utilized to assess factors

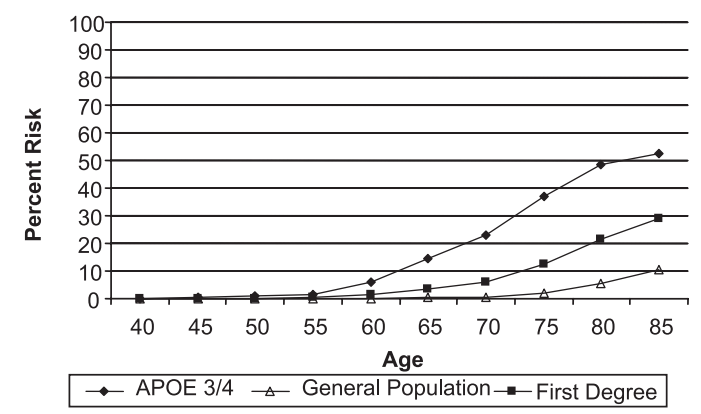

Fig. 1. Example of an Alzheimer's disease (AD) risk curve presented to participants. associated with accurate recall of both types of information (genotype and lifetime risk estimates) at six weeks and one year; variables assessed included gender, education, number of relatives affected with $\mathrm{AD}$, and baseline worry about developing $\mathrm{AD}$.

\section{RESULTS}

Demographic characteristics of the study sample $(\mathrm{N}=104)$ and genotype breakdown are shown in Table 1. All participants underwent neuropsychological screening and formal memory testing was within normal limits for all participants. Descriptive statistics indicate that at the six-week follow-up, 61 participants (59\%) accurately recalled the numeric risk probability they were given, 24 (23\%) incorrectly recalled their risk and 19 $(18 \%)$ indicated that they did not remember the information. Of the 24 individuals who did not accurately recall their numeric risk probability, 7 (29\%) overestimated and 17 (71\%) underestimated their risk. Seventy-two (69\%) correctly recalled their APOE genotype, 13 (13\%) incorrectly recalled their genotype, and $19(18 \%)$ did not remember the information (Table 2).

At the one-year follow-up, 50 participants (48\%) accurately recalled the numeric risk probability they were given, 37 (36\%) did not accurately recall their risk, and 17 (16\%) indicated that they did not remember the information. Of the 37 individuals who did not accurately recall their numeric risk probability, 12 (32\%) overestimated and $25(68 \%)$ underestimated their risk. Sixty-three participants $(63 \%)$ correctly recalled their $A P O E$ genotype, $10(10 \%)$ were incorrect in their recall of genotype and $28(28 \%)$ indicated they did not remember (Table 2). A detailed breakdown of correct recall of lifetime risk for 6 weeks and 12 months post-disclosure shows that $59 \%$ of the participants were correct within 5 points of the risk estimate given and $71 \%$ were correct within 15 points of the risk estimate given (Table 3). Although it was not a focus of the study, an analysis of the Control Arm recall showed that there was no significant difference for accurate lifetime risk recall between the Intervention Arm and the Control Arm.

At the one-year follow-up, all participants were asked whether they knew the risk-enhancing form of APOE. Sixty-

Table 1

Demographic characteristics of the sample $(\mathrm{N}=104)$

\begin{tabular}{lc}
\hline Mean Age, years (SD) & $51.3(9.7)$ \\
Gender (F/M) & $73 / 31$ \\
Mean years education (SD) & $16.6(2.1)$ \\
APOE genotype, N (\%) & \\
$3 / 3$ & $51(49)$ \\
$3 / 4$ & $43(41)$ \\
$2 / 3$ & $4(4)$ \\
$4 / 4$ & $3(3)$ \\
$2 / 4$ & $3(3)$ \\
\hline
\end{tabular}


Table 2

Number of participants accurately recalling lifetime risk estimate, APOE genotype and possession of risk-enhancing gene ( $\varepsilon 4$ allele)

\begin{tabular}{lcc}
\hline & \multicolumn{2}{c}{ Number correct $(\%)$} \\
\cline { 2 - 3 } & 6 Weeks & 1 Year \\
\hline Lifetime risk estimate & $\mathrm{N}=104)$ & $50(48 \%)$ \\
Accurate & $61(59 \%)$ & $37(36 \%)$ \\
Incorrect & $24(23 \%)$ & $17(16 \%)$ \\
Didn't recall & $19(18 \%)$ & $(\mathrm{N}=100)^{a}$ \\
Genotype & $(\mathrm{N}=104)$ & $63(63 \%)$ \\
Accurate & $72(69 \%)$ & $10(10 \%)$ \\
Incorrect & $13(13 \%)$ & $28(28 \%)$ \\
Didn't recall & $19(18 \%)$ & $(\mathrm{N}=100)^{a}$ \\
Possession of $\varepsilon 4$ allele & $\mathrm{N} / \mathrm{A}^{b}$ & $76(76 \%)$ \\
\hline
\end{tabular}

${ }^{a}$ Missing data.

${ }^{b}$ Participants were not asked this question at this timepoint.

Table 3

Participant recall of lifetime risk $(\mathrm{N}=104)$

\begin{tabular}{lcc}
\hline Lifetime risk recall & $\begin{array}{c}\text { 6 Week post-disclosure } \\
\text { number }(\%)\end{array}$ & $\begin{array}{c}1 \text { Year post-disclosure } \\
\text { number }(\%)\end{array}$ \\
\hline Exact & $28(27 \%)$ & $20(19 \%)$ \\
Within 5 points & $33(32 \%)$ & $30(29 \%)$ \\
$\begin{array}{l}\text { Within } 6 \text { to } 10 \\
\text { points }\end{array}$ & $7(7 \%)$ & $10(10 \%)$ \\
$\begin{array}{l}\text { Within } 11 \text { to } 15 \\
\text { points }\end{array}$ & $6(6 \%)$ & $3(11 \%)$ \\
$\begin{array}{l}\text { Within } 16 \text { to } 20 \\
\text { points } \\
>20 \text { points }\end{array}$ & $5(5 \%)$ & $13(12 \%)$ \\
Don't remember & $6(6 \%)$ & $17(16 \%)$ \\
\hline
\end{tabular}

Accurate recall defined in text as within $5 \%$ of the numeric risk estimate disclosed to participants.

four participants (62\%) correctly indicated that $\varepsilon 4$ is the riskenhancing $A P O E$ gene. All participants were also asked whether they carried the "risk-enhancing form of APOE." The question was worded so that individuals would not be required to know that the $\varepsilon 4$ allele is the risk-enhancing form, but only whether they possessed zero, one or two copies of the gene that increased AD risk. Seventy-six participants (76\%) correctly recalled how many copies of the risk-enhancing allele they possessed. There were three $\varepsilon 4$ homozygotes in the study sample and all three correctly recalled that they possessed two copies of the risk gene.

McNemar's $\chi^{2}$ analyses revealed that at one year after disclosure, participants were significantly more likely to recall their specific genotype than their numeric risk estimate (Table $2 ; P<0.05)$. No difference was seen at six weeks. $\chi^{2}$ analyses demonstrated that participants who were $\varepsilon 4$-positive were more likely to correctly recall their genotype at 6 weeks $(P<$ $0.05)$ and one year $(P<0.05)$ than those participants who were $\varepsilon 4$-negative. $\chi^{2}$ analyses also showed that participants were significantly more likely to recall whether they possessed a copy of the risk gene than to recall their specific genotype $(P<0.05)$. There was a trend suggesting that participants who were worried about developing $\mathrm{AD}$ at baseline were more likely to recall their genotype at six weeks $(P=0.07)$, but this association was not apparent at one year. Logistic regression revealed that $A P O E$ genotype predicted accurate recall of genotype information $(P<0.05)$ with $\varepsilon 4$-positive participants more likely to recall their genotype than $\varepsilon 4$-negative individuals one year after disclosure. None of the other study variables entered into the model (gender, education, baseline AD worry, and number of affected relatives) significantly contributed to genotype recall. No significant predictors were found for recall of lifetime risk at one year or for either type of information at six weeks.

\section{DISCUSSION}

This study is the first to examine whether participants correctly recall their APOE genotype and numeric risk estimates after undergoing a risk assessment for $\mathrm{AD}$. Genetic counseling studies have shown that accurate recall of numeric risk estimates for cancer vary widely with a range of $31 \%{ }^{24}$ to $81 \%,{ }^{14}$ although a lack of standardization in how risk was presented (odds or percentages) and in how recall accuracy was defined likely contributed to this large range. ${ }^{25}$ Our data fall within this previously reported range with 59\% recall accuracy of the numeric risk estimate (defined as within 5\%) at six weeks postdisclosure and $48 \%$ accuracy at one year. Our study is also unique in that we were able to analyze recall of genetic test results because there are six possible genotypes for APOE as opposed to either a mutation positive or negative result in genetic testing for hereditary cancer.

In our study, a sizable number of people could not remember or did not accurately recall risk information. Six weeks after the risk assessment and disclosure, $41 \%$ of the participants did not accurately recall their risk estimate and $31 \%$ did not accurately recall their genotype. Inaccurate recall increased at one year post-disclosure when $52 \%$ of the participants could not correctly recall their risk estimate and $47 \%$ could not accurately recall their genotype. In addition, at the one-year follow-up $38 \%$ of the participants were not able to identify which $A P O E$ allele increased risk and $24 \%$ of participants were not able to recall how many copies of the risk-enhancing gene they possessed. A primary reason for the poor recall may have been information overload. During the disclosure session, the general population lifetime risk, the risk to all first-degree relatives of $\mathrm{AD}$ patients, genotype specific lifetime risk, remaining risk, and $A P O E$ genotype were presented; this amount of information may have been confusing or difficult for an individual to process and recall. Also, as in genetic counseling for cancer, the information presented in $\mathrm{AD}$ genetic counseling is complex. For example, to stress the fact that $\mathrm{AD}$ is multifactorial and that age is a risk factor, we presented prevalence statistics showing 
that $50-60 \%$ of people in their nineties suffer from dementia, most of which is $\mathrm{AD}$. It is possible that some study participants may have confused such general information with their own personal risk information. Alternatively, the general information may have played a role in how an individual perceived and recalled his or her personal risk, such as believing his or her lifetime risk was $50 \%$ because approximately $50 \%$ of individuals in their nineties suffer from AD. Previous studies have described the intermingling of general information and personal risk information ${ }^{26}$ and credited it to the large amount of information presented during genetic counseling sessions. ${ }^{15}$ In the future, it may be worthwhile to simplify the general information presented with a handout prior to individualized counseling to allow the counselor to spend less time on formal education and more time on interpersonal counseling.

Several previous studies have reported that participants who do not accurately recall their risk tend to overestimate ${ }^{15-17,27}$ and that overestimation has been associated with worry about developing the disease. ${ }^{17,28-30}$ However, we did not observe these phenomena among the participants in our study. Of the participants who did not accurately recall their risk, $71 \%$ and $68 \%$ underestimated their risk at six weeks and one year postdisclosure, respectively. It is possible that this underestimation served as a defense against potentially threatening information, which has been described in other health behavior studies. ${ }^{31,32}$ Although there was a trend toward significance in the association between worry at baseline and correct recall of risk at six weeks, there was no such association between baseline worry and recall of risk information at the one-year follow-up.

Our findings suggest that APOE genotype, specifically possession of an $\varepsilon 4$ allele, may be more memorable than numeric estimates of lifetime risk of $\mathrm{AD}$. At one year after disclosure, participants were significantly more likely to recall their specific genotype compared to the risk estimate and $\varepsilon 4$ positive individuals were significantly more likely to recall their genotype six weeks and one year after disclosure. The influence and impact of disclosing $A P O E$ genotype as part of an $\mathrm{AD}$ risk assessment has also been observed in other REVEAL studies examining the effect of genetic test result disclosure on risk perception. ${ }^{8,9}$

There are several possible explanations for the association between $A P O E$ genotype disclosure and accurate information recall. A methodological consideration is that participants were asked about $A P O E$ genotype recall in a multiple choice format while they were asked about lifetime risk in a more challenging open-ended format. The nature of the risk information itself may have also influenced recall. Study participants may have been motivated to participate in the study because of the potential of receiving genetic test results and thus may have focused more on APOE genotype, especially the possession of the allele that increases $\mathrm{AD}$ risk. The way that the numeric risk estimate was presented may have also contributed to poorer recall of this information. Participants were informed that the numeric risk estimates were based on population studies, thus APOE genotype may have been perceived as more personalized and more memorable information when compared to the risk estimate. In addition, the risk estimate was presented as a probability and the range of possible risk estimates was $13-57 \%$. Therefore, the risk estimate was midrange and typically less than the usual probabilities associated with genetic testing, specifically Mendelian disorders. The study participants, despite their high education level, may have been uncomfortable with mathematical concepts and probabilities, which have been reported elsewhere. ${ }^{32-34}$ Also, it is possible that some participants might have shown better recall if the risk estimates had been presented in a different format, such as odds or in a more descriptive and discrete format, such as high or low. ${ }^{16,33}$

Participants were significantly more likely to remember the more general information of whether they carried the risk gene than to recall their specific genotype. These findings support previously documented observations that individuals often dichotomize risk and thus perceive the risk in a binary form, such as "I will or won't get the disease," or "I do or don't possess the risk gene." 35 These findings as well as the fact that $59 \%$ of participants recalled the risk estimate within 5 percentage points and $71 \%$ of participants recalled lifetime risk within 15 percentage points of the actual risk disclosed (Table 3 ) are also congruous with the belief that individuals simplify complex information and extract the gist. ${ }^{7,36,37}$ Although it is beneficial for an individual undergoing genetic testing and counseling for a complex disorder to correctly recall the essential information communicated, especially if preventative measures are identified, it is also important that they understand that the risk is probabilistic. A probabilistic risk of developing $\mathrm{AD}$ may be an important tool in deciding whether an at-risk individual should undergo preventative measures that have side effects and more studies are needed to develop effective ways to communicate the concept of probabilities.

There are some limitations to this study. Our participants are not representative of the general population at risk for $\mathrm{AD}$, given that they are predominantly White, female, and have a high level of education. Although the education session presentation was standardized, the questions asked during the education session as well as the content of the individualized genetic counseling sessions both before and during disclosure were appropriately not standardized. In addition, the genetic counselors likely had different counseling styles and may have emphasized different issues in accordance with the questions and concerns of the participants. It is possible that the accuracy of information recall and risk perception also reflects the skills of the health professional providing the counseling, but studies have been inconclusive on this point. ${ }^{38,39}$ Future REVEAL studies will analyze the individual counseling sessions and relate counseling process variables to various outcomes such as risk perception and recall. In addition, the participants in REVEAL will be followed for an additional eight years beyond the disclosure session which will allow us to assess risk information recall and perception along with other variables over a much longer period.

This is the first study to assess information recall in genetic counseling and testing for late-onset Alzheimer's disease. Not only are the findings significant for risk communication in $\mathrm{AD}$, 
they are also relevant for cancer counseling and for genetic counseling for other complex diseases, especially as more susceptibility genes and effective preventative strategies are found. The data imply that individuals are more likely to accurately recall discrete information, such as genotype, than probabilistic information, such as the risk estimate. Furthermore, participants recalled the genotype information in a more simplified format than it was presented. Considering the complexity, magnitude, and probabilistic nature of the information presented in $\mathrm{AD}$ genetic counseling, additional research identifying effective risk communication is clearly needed.

\section{ACKNOWLEDGMENTS}

This study was supported by National Institute of Health grants RO1 HG/AG 02213 (REVEAL study), P30 AG13846 (Boston University Alzheimer Disease Center) and M01 RR00533 (Boston University General Clinical Research Center).

\section{References}

1. Green RC. Diagnosis and management of Alzheimer's disease and other dementias, 2nd edition. Caddo: Professional Communications, Inc., 2005.

2. Lendon CL, Ashall F, Goate AM. Exploring the etiology of Alzheimer disease using molecular genetics. JAMA 1997;227:825-831.

3. Strittmatter WJ, Saunders AM, Schmechel D, Pericak-Vance M, et al. Apolipoprotein E: high-avidity binding to beta-amyloid and increased frequency of type 4 allele in late-onset familial Alzheimer disease. Proc Natl Acad Sci U S A 1993;90:19771981.

4. Brodaty H, Conneally M, Gauthier S, Jennings C, et al. Consensus statement on predictive testing for Alzheimer disease. Alzheimer Dis Assoc Disord 1995;9:182-187.

5. Statement on use of Apolipoprotein E testing for Alzheimer disease. American College of Medical Genetics/ American Society of Hum Genet Working Group on Apo E and Alzheimer disease [Consensus Development Conference] JAMA 1995;274: 1627-1629.

6. Apolipoprotein E genotyping in Alzheimer's disease. National Institute on Aging/ Alzheimer's Association Working Group [Consensus Development Conference, NIH] Lancet 1996;347:1091-1095.

7. Burke W, Pinsky LE, Press NA. Categorizing Genetic Tests to Identify Their Ethical, Legal, and Social Implications. Am J Med Genet 2001;106:233-240.

8. Marteau TM, Roberts S, LaRusse S, Green RC. Predictive Genetic Testing for Alzheimer's Disease: Impact upon Risk Perception. Risk Anal 2005;25:397-404.

9. LaRusse S, Roberts JS, Marteau TM, Katzen H, et al. Genetic susceptibility testing versus family history-based risk assessment: Impact on perceived risk of Alzheimer disease. Genet Med 2005;7:48-53.

10. Clarke A, Parsons E, Williams A. Outcomes and process in genetic counselling. Clin Genet 1996;50:462-469.

11. Evans DG, Blair V, Greenhalgh R, Hopwood P, et al. The impact of genetic counselling on risk perception in women with a family history of breast cancer. $\mathrm{BrJ}$ Cancer 1994;70:934-938.

12. Meiser B, Halliday JL. What is the impact of genetic counselling in women at increased risk of developing hereditary breast cancer? A meta-analytic review. Soc Sci Med 2002;54:1463-1470.

13. van Dijk S, Otten W, van Asperen CJ, Timmermans DR, et al. Feeling at risk: how women interpret their familial breast cancer risk. Am J Med Genet 2004;131:A42A49.

14. Cull A, Miller H, Porterfield T, Mackay J, et al. The use of videotaped information in cancer genetic counselling: a randomized evaluation study. Br J Cancer 1998;77:830 837.
15. Watson M, Lloyd S, Davidson J, Meyer L, et al. The impact of genetic counseling on risk perception and mental health in women with a family history of breast cancer. Br J Cancer 1999;79:868-874.

16. Lobb EA, Butow PN, Meiser B, Barratt A, et al. Women's preferences and consultants' communication of risk in consultations about familial breast cancer: impact on patient outcomes. J Med Genet 2003;40:E56.

17. Gurmankin $\mathrm{AD}$, Baron J, Armstrong K. Intended message versus message received in hypothetical physician risk communications: exploring the gap. Risk Anal 2004; 24:1337-1347.

18. Hopwood P, Keeling F, Long A, Pool C, et al. Psychological support needs for women at high genetic risk of breast cancer: some preliminary indicators. Psychooncology 1998;7:402-412.

19. Audrain J, Lerman C, Rimer B, Cella D, et al. Awareness of heightened breast cancer risk among first degree relatives of recently diagnosed breast cancer patients. Cancer Epidemiol Biomarkers Prev 1995;4:561-565.

20. Roberts JS, LaRusse SA, Katzen H, Whitehouse PJ, et al. Reasons for seeking genetic susceptibility testing among first-degree relatives of people with Alzheimer disease. Alzheimer Dis Assoc Disord 2003;17:86-93.

21. Cupples LA, Farrer LA, Sadovnick AD, Relkin N, et al. Estimating risk curves for first-degree relatives of patients with Alzheimer's disease: the REVEAL study. Genet Med 2004;6:192-196.

22. Randolph C, Tierney MC, Mohr E, Chase TN The Repeatable Battery for the Assessment of Neuropsychological Status (RBANS): preliminary clinical validity. J Clin Exp Neuropsychol 1998;20:310-319.

23. Lerman C, Lustbader E, Rimer B, Daly M, et al. Effects of individualized breast cancer risk counseling: a randomized trial. J Natl Cancer Inst 1995;87:286-292.

24. Watson M, Lloyd S, Davidson J, Meyer L, et al. The impact of genetic counselling on risk perception and mental health in women with a family history of breast cancer. Br J Cancer 1999;79:868-874.

25. van Dijk S, Otten W, van Asperen CJ, Timmermans DR, et al. Feeling at risk: how women interpret their familial breast cancer risk. Am J Med Genet 2004;131:A42A49.

26. Parsons E, Atkinson P. Lay constructions of genetic risk. Sociol Health Illn 1999;14: $437-455$.

27. Gurmankin AD, Domchek S, Stopfer J, Fels C, et al. Patients' resistance to risk information in genetic counseling for BRCA1/2. Arch Intern Med 2005;165:523-529.

28. Black WC, Nease RF, Tosteson AN. Perceptions of breast cancer risk and screening effectiveness in women younger than 50 years of age. J Natl Cancer Inst 1995;87:720 731.

29. Webb JA, Friedman LC, Bruce SB, Weinberg AD, et al. Demographic, psychosocial, and objective risk factors related to perceived risk of skin cancer. J Cancer Educ 1996;11:174-177.

30. Baron J, Hershey JC, Kunreuther H. Determinants of priority for risk reduction: the role of worry. Risk Anal 2000;20:413-427.

31. Croyle RT, Sun YC, Louie DH. Psychological minimization of cholesterol test results: moderators of appraisal in college students and community residents. Health Psychol 1993;12:503-507.

32. Cameron LD, Leventhal H. The Self-Regulation of Health and Illness Behaviour. London: Routledge; 2003.

33. Chase GA, Faden RR, Holtzman NA, Chwalow AJ, et al. Assessment of risk by pregnant women: implications for genetic counseling and education. Soc Biol 1986; 33:57-64.

34. Lipkus I, Samsa G, Rimer B. General performance on a numeracy scale among highly educated samples. Med Decis Making 2001;21:37-44.

35. Harper PS. What do we mean by genetic testing? J Med Genet 1997;34:749-752.

36. Lippman-Hand A, Fraser FC. Genetic counseling: provision and reception of information. Am J Med Genet 1979;3:113-127.

37. Reyna VF, Brainerd CJ. Fuzzy-trace theory and framing effects in choice: gist extraction, truncation and conversion. J Behav Decis Making 1991;4:249-262.

38. Julian-Reynier C, Welkenhuysen M, Hagoel L, Decruyenaere M, et al. Risk communication strategies: state of the art and effectiveness in the context of cancer genetic services. Eur J Hum Genet 2003;11:725-736.

39. Lloyd S, Watson M, Waites B, Meyer L, et al. Familial breast cancer: a controlled study of risk perception, psychological morbidity and health beliefs in women attending for genetic counselling. Br J Cancer 1996;74:482-487. 